\title{
Acknowledgment to Reviewers of Applied Microbiology in 2021
}

\author{
Applied Microbiology Editorial Office
}

MDPI AG, St. Alban-Anlage 66, 4052 Basel, Switzerland

Rigorous peer-reviews are the basis of high-quality academic publishing. Thanks to the great efforts of our reviewers, Applied Microbiology was able to maintain its standards for the high quality of its published papers. Thanks to the contribution of our reviewers, in 2021, the median time to first decision was 20.5 days and the median time to publication was 46 days. The editors would like to extend their gratitude and recognition to the following reviewers for their precious time and dedication, regardless of whether the papers they reviewed were finally published:

Adam Kawalek

Agnieszka Richert

Akiko Ogawa

Aleksandra Kocot

Alexa Ersilia

Alexander Paukov

Alina Pruss

Amisha T. Poret-Peterson

Anastasiia K. Kimeklis

András Bánvölgyi

Andrea Cortegiani

Andreas Beck

Andrew D. Cartmill

Andrey A. Belov

Ángel Emilio Martínez De Alba

Anna Cieślińska

Anna Jarosz-Wilkołazka

Anna Szczerba-Turek

Antonella Canini

Ashutosh P. Dubey

Astrid Mayr

Babak Pakbin

Bastian Hornung

Beniamino Cenci Goga

Blaženka Kos

Catherine A. O'neill

Chichun Ho

Corneliu Tanase

Daniel Schindler

Danuta Drozdowska

Darija Lušić Vukić

Dariusz Kokoszyński

Davor Želježić

Devin Stauff
Dhananjaya Kulkarni

Dorota Górniak

Dorota Papciak

Edyta Adamska

Eleonora Nicolai

Enrico Novelli

Esin Semih

Evelio Ramirez Miquet

Ewa Dudzińska

Farkas Edit

Fátima Milhano Santos

Filip Šupljika

Franklin Wang-Ngai Chow

Giovanni Gallo

Giulia Pignataro

Hae Kyung Lee

Hasan Ejaz

Hasan Nazik

Horacio Bach

Hourei Oh

Igor Łoniewski

Ioannis A. Giantsis

Ivan Krešimir Svetec

Jae-Ho Shin

Jan Bobek

Joanna Kaminska

John O'Hagan

Jonathan Butler

Karel Sedlar

Kateřina Petř́čcková

Kathryn Kauffman

Kirill Antonets

Ki-Sun Yoon

Kristie Tanner 
Lara Vimercatti

Leontina C. Gurgu

Louisa Ludwig-Begall

Mara Gyöngyvér

Maria Alexandrovna Lebedeva

Maria Pilar Cortés

Mariusz Cycoń

Masataka Oda

Matteo Calcagnile

Matthias Eder

Michela Verni

Mohit Bibra

Myron Christodoulides

Nicoleta Ungureanu

Nishida Takehiro

Obed Hernandez-Gomez

Patrícia Branco

Patryk Tarka

Pieter Van Dillewijn

Przemyslaw Siejak

Ramachandran Chelliah

Renata Tisi

Rianna Murray
Robert D. Roghair

Roberto Fernandez-Lafuente

Rosa Alduina

Rositsa Stefanova Denkova-Kostova

Saad El-Din Hassan

Sabina Purkrtová

Sabrina Cadel-Six

Sanjay Swarup

Sanna Sillankorva

Sara Linden

Sebastian Przemieniecki

Serajus Salaheen

Seung Wook Kim

Sophie Blat

Stanisław Pietr

Stanley Brul

Stefan Shilev

Tatiana Korshunova

Vojtěch Sedláček

Wendy Mok

Wendy Stone

Xuan $\mathrm{Mu}$

Ying Chen Lu 